\title{
Capacity of a LoRaWAN Cell
}

\author{
Martin Heusse \\ Martin.Heusse@imag.fr \\ Univ. Grenoble Alpes, CNRS, \\ Grenoble INP, LIG \\ Grenoble, France
}

\author{
Takwa Attia \\ Takwa.Attia@univ-grenoble-alpes.fr \\ Univ. Grenoble Alpes, CNRS, \\ Grenoble INP, LIG \\ Grenoble, France
}

\author{
Christelle Caillouet \\ Christelle.Caillouet@univ- \\ cotedazur.fr \\ Univ. Côte d'Azur, I3S Inria \\ Sophia Antipolis, France
}

\author{
Franck Rousseau \\ Franck.Rousseau@imag.fr \\ Univ. Grenoble Alpes, CNRS, \\ Grenoble INP, LIG \\ Grenoble, France
}

\author{
Andrzej Duda \\ Andrzej.Duda@imag.fr \\ Univ. Grenoble Alpes, CNRS, \\ Grenoble INP, LIG \\ Grenoble, France
}

\begin{abstract}
In this paper, we consider the problem of evaluating the capacity of a LoRaWAN cell. Previous analytical studies investigated LoRaWAN performance in terms of the Packet Delivery Ratio (PDR) given a number of devices around a gateway and its range. We improve the model for PDR by taking into consideration that the following two events are dependent: successful capture during a collision and successful frame decoding despite ambient noise. We consider a realistic traffic model in which all devices generate packets with the same inter-transmission times corresponding to the duty cycle limitation at the highest SF, regardless of the distance to the gateway. Based on the developed model, we optimize the Spreading Factor (SF) boundaries to even out PDR throughout the cell. We validate the analytical results with simulations, compare our model with previous work, and experimentally validate the hypothesis of Rayleigh fading for the LoRa channel. The important conclusion from our results is that a LoRa cell can handle a relatively large number of devices. We also show that there is practically no inter-SF interference (cross interference between transmissions with different SFs): interference from higher SFs comes from nodes located farther away, so they face greater attenuation and thus, they do not interfere with lower SF nodes.
\end{abstract}

\section{CCS CONCEPTS}

- Networks $\rightarrow$ Network performance modeling; Very long-range networks; Sensor networks.

\section{KEYWORDS}

LoRaWAN; Packet Delivery Ratio; capacity; modeling

\section{ACM Reference Format:}

Martin Heusse, Takwa Attia, Christelle Caillouet, Franck Rousseau, and Andrzej Duda. 2020. Capacity of a LoRaWAN Cell. In 23rd International ACM Conference on Modeling, Analysis and Simulation of Wireless and Mobile

Publication rights licensed to ACM. ACM acknowledges that this contribution was authored or co-authored by an employee, contractor or affiliate of a national government. As such, the Government retains a nonexclusive, royalty-free right to publish or reproduce this article, or to allow others to do so, for Government purposes only. MSWiM '20, November 16-20, 2020, Alicante, Spain

(C) 2020 Copyright held by the owner/author(s). Publication rights licensed to ACM ACM ISBN 978-1-4503-8117-8/20/11 ..\$15.00

https://doi.org/10.1145/3416010.3423228
Systems (MSWiM '20), November 16-20, 2020, Alicante, Spain. ACM, New York, NY, USA, 10 pages. https://doi.org/10.1145/3416010.3423228

\section{INTRODUCTION}

In this paper, we consider the problem of evaluating the capacity of a LoRaWAN cell. LoRaWAN defines a physical layer based on Chirp Spread Spectrum (CSS) for robust communication in the sub-GHz ISM band. The data rate depends on the Spreading Factor (SF) that characterizes the number of bits carried by a chirp. SF allows to trade data rate for range: a small value of SF (e.g., 7) results in a higher data rate, short transmissions, and a small range, while SF12 gives the best sensitivity and the longest range, at the cost of long transmission times and worst energy consumption.

LoRa devices use a channel access method similar to unslotted ALOHA [1]: a device may wake up at any instant and start transmitting a packet to the LoRa gateway without testing for on-going transmissions. The performance in terms of the Packet Delivery Ratio (PDR) and scalability strongly depend on the number of collisions arising when at least two end devices transmit packets overlapping in time. Transmissions using different spreading factors are quasi-orthogonal-in case of a collision, both frame transmissions succeed if one is not several orders of magnitude stronger than the other. For collisions of transmissions with the same SF, a frame transmission succeeds if it is significantly stronger than the other one, a phenomenon called the capture effect ${ }^{1}$.

For an increasing distance to the gateway, a device needs to change SF to maintain the desired level of PDR: farther from the gateway, the nodes need to use larger SF to increase the probability of frame reception. We can thus define SF boundaries around the gateway in form of concentric annuli in which devices share the same value of SF. In practice, the Adaptive Data Rate (ADR) commands issued by the LoRa network server give SF to use based on the measured SNR (Signal to Noise Ratio) and PDR at the gateway. Larger SFs result in more robust transmissions by using a lower data rate with similar transmission power. However, transmissions with larger SF take more time, which increases contention and collision probability.

Previous analytical studies investigated the LoRaWAN performance in terms of PDR given a number of devices around a gateway

\footnotetext{
${ }^{1}$ The capture effect refers to the capacity of correctly receiving a significant fraction of colliding frames [2-4].
} 
and its range [5-10]. In this paper, we bring the following contributions:

- We propose a traffic model in which all devices generate packets with the same inter-transmission times corresponding to the duty cycle limitation at the highest SF regardless of the distance to the gateway (Section 3).

- We develop a simple yet precise model for PDR assuming that the following two events are dependent: successful capture during a collision and successful frame decoding despite ambient noise (Section 4).

- We validate the analytical results with discrete event simulations and compare with models proposed in the literature in Section 5.

- We evaluate, by simulation as well as analytically, the capacity of a LoRaWAN cell for a given density of nodes per $\mathrm{km}^{2}$ and given SF boundaries. We first study the case of boundaries located at distances corresponding to predetermined average SNR levels. Second, we optimize the SF boundaries so that the maximal number of nodes obtain given target PDR (Section 6).

- We experimentally validate the hypothesis of Rayleigh fading for the LoRa channel model by analyzing the distribution of channel gains measured on a real-world testbed (Section 7).

The important conclusion from our results is that a LoRa cell can handle a large number of devices, e.g., about 900 nodes can get at least PDR of $90 \%$ in a $10 \mathrm{~km}^{2}$ cell or more than 1550 nodes can get $60 \%$ PDR in a $78 \mathrm{~km}^{2}$ cell. We also show that the traffic pattern that we use leads to practically no inter-SF interference (cross interference between transmissions with different SFs).

We start the paper with some background on LoRa in Section 2. We end it with the discussion of previous work in Section 8 and conclusions in Section 10.

\section{LORAWAN BASICS AND ASSUMPTIONS}

The physical layer of LoRaWAN has the following parameters [11] Bandwidth (BW) - the range of the frequency sweep of CSS, between $7.8 \mathrm{kHz}$ and $500 \mathrm{kHz}$. Larger bandwidth results in a higher data rate, but lowers the sensitivity.

Spreading Factor (SF) SF varies between 6 (7 in practice) and 12, with SF12 resulting in the best sensitivity and range, at the cost of the lowest data rate and worst energy consumption. Decreasing SF by 1 unit roughly doubles the transmission rate and divides by 2 the transmission duration as well as energy consumption.

Coding Rate (CR) - the rate of Forward Error Correction (FEC) that improves packet error rate in presence of noise and interference. A lower coding rate results in better robustness, but increases the transmission time and energy consumption. The possible values are: $4 / 5,4 / 6,4 / 7$, and $4 / 8$.

Transmission Power $(P)$ : LoRaWAN defines the following values of $P$ for the EU $863-870 \mathrm{MHz}$ band: 2, 5, 8, 11, and $14 \mathrm{dBm}$.

Table 1 presents $\mathrm{SF} j$, data rate $\mathrm{DR} j$, Signal-to-Noise Ratio (SNR) limit, airtime $\tau_{j}$, and $s_{\text {max }}$, the maximum payload size. $\tau_{j}$ denotes the transmission duration of the maximum size frame at data rate DRj.
Table 1: LoRa parameters for BW of $125 \mathrm{kHz}$.

\begin{tabular}{rrrrr} 
SFj & SNR limit $\left(q_{j}\right)$ & Airtime $\left(\tau_{j}\right)$ & Bit rate DRj & $s_{\max }$ \\
\hline 7 & $-7.5 \mathrm{~dB}$ & $102.7 \mathrm{~ms}$ & DR5: $5469 \mathrm{~b} / \mathrm{s}$ & $230 \mathrm{~B}$ \\
8 & $-10 \mathrm{~dB}$ & $184.8 \mathrm{~ms}$ & DR4: $3125 \mathrm{~b} / \mathrm{s}$ & $230 \mathrm{~B}$ \\
9 & $-12.5 \mathrm{~dB}$ & $328.7 \mathrm{~ms}$ & DR3: $1758 \mathrm{~b} / \mathrm{s}$ & $123 \mathrm{~B}$ \\
10 & $-15 \mathrm{~dB}$ & $616.5 \mathrm{~ms}$ & DR2: $977 \mathrm{~b} / \mathrm{s}$ & $59 \mathrm{~B}$ \\
11 & $-17.5 \mathrm{~dB}$ & $1315 \mathrm{~ms}$ & DR1: $537 \mathrm{~b} / \mathrm{s}$ & $59 \mathrm{~B}$ \\
12 & $-20 \mathrm{~dB}$ & $2466 \mathrm{~ms}$ & DR0: $293 \mathrm{~b} / \mathrm{s}$ & $59 \mathrm{~B}$ \\
\hline
\end{tabular}

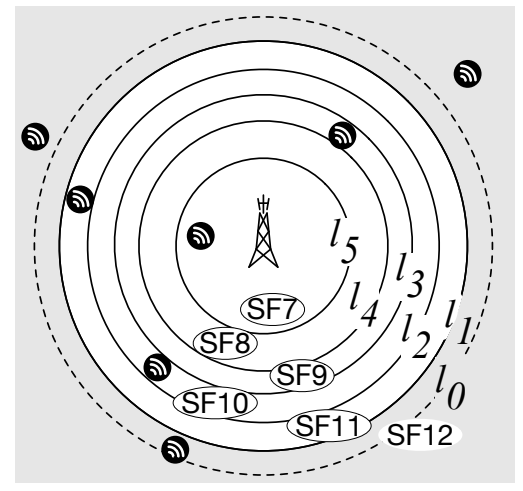

Figure 1: Annuli of SF allocation around the gateway

Table 2: Notation

\begin{tabular}{lc}
\hline Spatial density of nodes & $\rho$ \\
Packet generation intensity & $\lambda_{t}$ \\
Frame transmission duration at data rate DRj & $\tau_{j}$ \\
Distance of farthest node using DRj & $l_{j}$ \\
Offered traffic (in Erlang) at DRj & $v_{j}$ \\
Average channel gain at distance $d$ & $g(d)$ \\
SNR threshold for DRj & $q_{j}$ \\
Power gap for successful capture (typically 6 dB) & $\gamma$ \\
Transmission power, In-band noise power & $P, N$ \\
Transmission success probability taking into account & \\
\multicolumn{1}{c}{ attenuation, fading, thermal noise } & $H$ \\
Transmission success probability due to collisions & $Q$ \\
\hline
\end{tabular}

We consider in our analysis the basic LoRa CSS modulations with $125 \mathrm{kHz}$ bandwidth and SF in 12,11,.. 7, which corresponds to data rates $\mathrm{DR} j$, with $j=0,1, \ldots 5$, and $\mathrm{SF}=12-j$ (see Table 1 ).

\subsection{Assumptions and Notation}

We consider a LoRaWAN cell with devices that configure SF based on their transmission conditions, which corresponds to the annuli view presented in Figure 1. $l_{j}$ denotes the distance to the farthest device that uses SF $\{12-j\}$ and thus data rate DRj. Outside $l_{1}$, there is no lower data rate available so all nodes need to use SF12. In this way, $l_{1}$ is the effective cell range to consider.

We use the Okumura-Hata model for path loss attenuation (also used in other work [12-14]), the suburban environment variant with an antenna height of $15 \mathrm{~m}$. This empirical model is slightly less favorable but more realistic than adopting an arbitrary path loss exponent as in most of previous work cited herein. We consider a gateway antenna gain of $6 \mathrm{~dB}$, which compensates for a receiver noise factor of $6 \mathrm{~dB}$. We neglect shadowing, since its net effect would be small in our case: it would modify the channel gain 
from each node without changing the general characteristics of the network [10]. Finally, we consider a Rayleigh channel: a multiplicative random variable with an exponential distribution of unit mean affects the received signal power.

\section{TRAFFIC MODEL}

We propose a traffic model in which all devices generate packets with the same inter-transmission times corresponding to the duty cycle limitation at the highest SF regardless of the distance to the gateway.

Such a model is more realistic than the assumptions of other work [5-10] in which nodes saturate their share of bandwidth whatever their position in the network, which means that nodes closer to the gateway generate more traffic than those farther away. In a LoRaWAN network, traffic is application driven and should not depend on the position of the node in the network or channel quality. An application needs to send a given number of bytes (limited by the maximal frame size) and its operation should be transparent with respect to the position of devices that will use the same data generation pattern irrespectively of the configured SF. Obviously, there are different IoT applications generating packets according to their needs, e.g., once a day, once an hour, under the constraint of the $1 \%$ duty cycle limit per band.

As devices operate with a given duty cycle and wake up to send data at constant intervals, the superposition of traffic coming from a large number of devices tends to a homogeneous Poisson process and we denote its intensity with $\lambda_{t}$ that depends on the data generation pattern of IoT applications. The limiting case is when $\lambda_{t}$ is the traffic intensity of devices operating at DR0 and SF12 at their maximal duty cycle and using $59 \mathrm{~B}$ packets, the maximum size at this rate. Our analysis will also hold for lower traffic intensity coming from less demanding applications.

We assume that devices are homogeneously scattered with spatial density $\rho$. The number of nodes using DR $j$ is a function of the radii defining the annulus between $l_{j+1}$ and $l_{j}$ around the gateway (see Figure 1). We assume that $l_{6}=0$, so DR6 and above are not used.

The duty cycle limitation is for each frequency band: LoRa devices have to limit their occupation of each frequency band to $1 \%$ of time with 3 to 5 frequency channels in each band. In Europe, for instance, there are 3 channels in band h1.4 and 5 in h1.3, so if we assume 3 channels, devices can use each of them at $0.33 \%$ duty cycle. The airtime of maximum size packets at DR0 corresponds to $\tau_{0}=2.466 \mathrm{~s}$, so they can be sent every $739.8 \mathrm{~s}$ on the same channel (or every $246.6 \mathrm{~s}$ on different channels) to achieve $0.33 \%$ duty cycle $\left(\frac{1}{300}\right)$ per channel. Thus,

$$
\lambda_{t}=\frac{1}{739.8 s} .
$$

For SF $=12-j$, and $n_{j}$, the number of nodes contending with each other in annulus $j$, offered traffic is

$$
v_{j}=n_{j} \tau_{j} \lambda_{t}
$$

in Erlang, where $\tau_{j}$ is the transmission duration at data rate $\mathrm{DR} j$, and

$$
n_{j}=\pi \rho\left(l_{j}^{2}-l_{j+1}^{2}\right)
$$

\section{MODEL FOR SUCCESSFUL PACKET DELIVERY}

We present below the model to compute PDR based on the notation in Table 2. First, we derive the expression for PDR under the assumption that collisions and successful overcoming the ambient noise are independent. Then, we relax this assumption and derive the final expression for PDR.

In a LoRaWAN cell, a given frame is not correctly received for two reasons (and maybe both): i) SNR is below the reception threshold and/or ii) a collision occurs and the signal is not strong enough relatively to the interference.

\subsection{Successful frame reception, no collisions}

Provided that there is no collision, a frame transmission succeeds as long as SNR at the receiver for this transmission is above $q_{j}$, the minimum SNR for the corresponding spreading factor [15]. The received signal power depends on the distance and Rayleigh fading, whereas the noise power is the constant thermal noise for a $125 \mathrm{kHz}$-wide band: $N=-123 \mathrm{dBm}(-174 \mathrm{dBm}$ per $\mathrm{Hz})$.

Thus, the probability of successful transmission from distance $d$ at data rate $\mathrm{DR} j$ is:

$$
H=\exp \left(-\frac{N q_{j}}{P g(d)}\right),
$$

where $g(d)$ is the average channel gain at distance $d[5]$.

\subsection{Successful frame reception, collisions}

For a given transmission attempt, provided that it has already met the signal strength reception condition (with probability $H$ ), we distinguish between three cases:

- Case 1 (0_collision) - if the transmission does not overlap any other frame, it succeeds;

- Case 2 (1_collision) - if a single frame transmitted on the same SF interferes with the transmission, then the frame is captured, if it is received with a power margin of at least $\gamma=6 \mathrm{~dB}$ against the colliding frame [15];

- Case 3 (2+collisions) - if two frames or more overlap the transmitted one, we simply deem it lost.

For a tagged transmission, the probability of Case 1 is:

$$
Q_{1}=\exp \left(-2 v_{j}\right) \text {, }
$$

as there should be no other transmission event during time $2 \tau_{j}$ to avoid overlap.

Case 2 (1_collision) happens when a single transmission occurs during time $2 \tau_{j}$ with probability $2 v_{j} \exp \left(-2 v_{j}\right)$ and the receiver successfully captures the frame. If we neglect the variability of $g(d)$ among the nodes using the same SF, then the received power in each annulus follows an exponential distribution. Successful frame capture occurs for a difference of $\gamma$ in received power (typically, $\gamma=6 \mathrm{~dB}$, which corresponds to a factor of 4). Since the probability that an exponential random variable is $\gamma$ times greater than another one is $(\gamma+1)^{-1}$, the probability of successful frame capture is just $(\gamma+1)^{-1}$. Thus, the probability of success in Case 2 is the following:

$$
Q_{2}=\frac{2}{\gamma+1} v_{j} \exp \left(-2 v_{j}\right) .
$$


For Case 3 (2+collisions), we can observe that collisions with successful packet capture against a single frame are already relatively rare, so meeting the capture condition for two interferers, or more, is effectively quite unlikely. Moreover, the co-occurrence of three transmissions is rare for manageable traffic intensity.

Thus, concurrent traffic impacts the successful packet reception with probability $Q$ :

$$
Q=Q_{1}+Q_{2}=\left(1+\frac{2}{\gamma+1} v_{j}\right) \exp \left(-2 v_{j}\right) .
$$

Finally, combining Eqs. 4 and 7 yields the probability of successful packet reception under the independence assumption:

$$
P D R_{I}=H \times Q .
$$

\subsection{Successful frame reception, dependent reception and capture}

In the model above, and in all the cited papers, the probability of capture in case of a collision and the probability of successfully overcoming the ambient noise are considered independent. However, it is not the case, as a strong signal at the receiver is at the same time more likely to overcome thermal noise and the interference from colliding transmissions.

In case of a single collision, the probability of successful frame reception at $\mathrm{DR} j$ is thus:

$$
\begin{aligned}
P D R_{1} & =\operatorname{Pr}\left(g_{r}>\frac{N q_{j}}{P g(d)} \cap g_{r}>\gamma g_{c}\right) \\
& =\operatorname{Pr}\left(g_{r}>\max \left(\frac{N q_{j}}{P g(d)}, \gamma g_{c}\right)\right),
\end{aligned}
$$

where $g_{r}$ and $g_{c}$ are the exponentially distributed Rayleigh channel gains for the considered frame and the colliding frame, $g(d)$ is the average path loss gain for both transmissions (thus assuming that both nodes are at distance $d$ from the gateway), and $\gamma$ is the required receiving power gap for successful capture (typically, $6 \mathrm{~dB}$ ).

If we denote $g_{t}=\frac{N q_{j}}{P g(d)}$, the threshold gain for successful reception against the thermal noise, the probability of successful frame reception becomes:

$$
\begin{aligned}
P D R_{1} & =\int_{0}^{+\infty} e^{-y} \int_{\max \left(g_{t}, \gamma y\right)}^{+\infty} e^{-x} \mathrm{~d} x \mathrm{~d} y \\
& =\frac{e^{-g_{t}}}{\gamma+1}\left(1+\gamma\left(1-e^{\frac{-g_{t}}{\gamma}}\right)\right)
\end{aligned}
$$

by integral of the probability density function over the successful reception interval.

Finally, the probability of successful packet reception under the dependence assumption is the following:

$$
P D R_{D}=H Q_{1}+2 v_{j} \exp \left(-2 v_{j}\right) P D R_{1} .
$$

\section{MODEL VALIDATION}

In this section, we compare our models and the model by Georgiou and Raza [5] with simulations.

\subsection{Simulations}

We have developed a discrete event simulator in Python for a single LoRa frequency channel used by a large number of nodes that takes

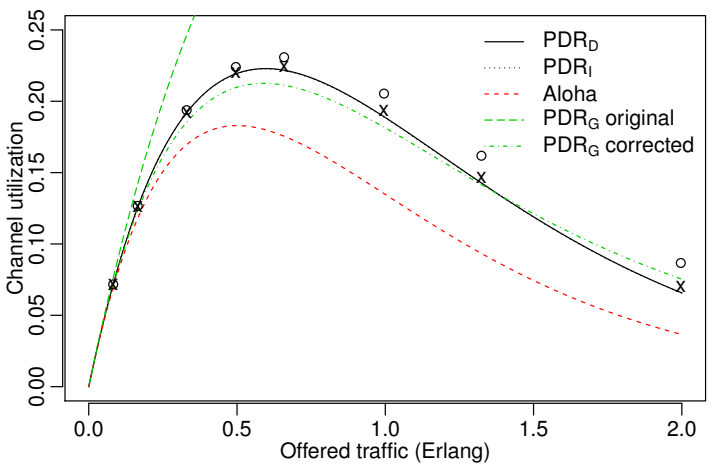

Figure 2: Channel utilization based on the values of $P D R_{I}$, $P D R_{D}$, and $P D R_{G}$ (the model by Georgiou and Raza [5], with or without correction) as well as the corresponding value for unslotted ALOHA, when all nodes are $2.5 \mathrm{~km}$ away from the gateway. Points correspond to the simulation values: o - success for $\gamma$ margin over the sum of the powers of all colliding frames; $x$ - success conditioned by at most one colliding frame and $\gamma$ margin.

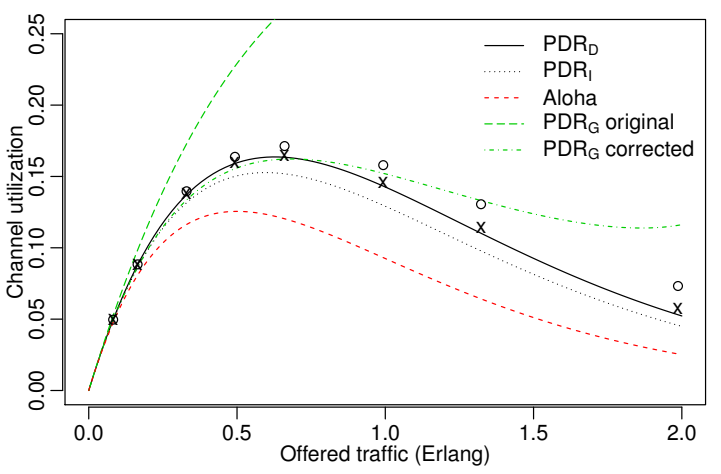

Figure 3: Channel utilization based on the values of $P D R_{I}$, $P D R_{D}$, and $P D R_{G}$ (the model by Georgiou and Raza [5], with or without correction) as well as the corresponding value for unslotted ALOHA, when all the nodes are $7.5 \mathrm{~km}$ away from the gateway.

into account Rayleigh fading, capture, as well as co-SF and inter$\mathrm{SF}$ interference ${ }^{2}$. It simulates the transmission of a large number of frames (e.g., $10^{7}$ ) generated by as many Poisson processes as $\mathrm{SF}$ zones, with intensities derived from the SF zone area, node density, and $\lambda_{t}$. The transmission distance distribution in each SF zone matches the node distribution. When frame transmissions overlap, the simulator computes co-SF and inter-SF interference to evaluate the effect of collisions, capture, and inter-SF jamming, using the theoretical [15] or experimental levels [16]. Based on the statistics of the number of correctly received frames, it computes the conditional PDR, given the distance to the gateway, with high precision (the confidence intervals are less than one percentage point). Compared to more complete simulators which include all

${ }^{2}$ https://gricad-gitlab.univ-grenoble-alpes.fr/heussem/lora-simple-discrete-eventsimulator 
protocol layers, focusing solely on contention allows to simulate hundred of thousand of frame transmission in a few minutes.

The simulator and analytical results corroborate each other. Simulation brings to light the effect of significant path loss differences between nodes in the same SF zone as well as the impact of inter-SF interference or the salience of the capture effect when multiple frames collide. Conversely, the analytical results are more suitable for SF boundary optimization that we develop in Section 6.

\subsection{Comparisons}

In this section, to compare our results with the familiar curves for the unslotted ALOHA model [1], we consider a variable number of nodes $n_{0}$, all at the same distance from the gateway. They send frames using SF12 with aggregate traffic intensity $v_{0}=n_{0} \tau_{0} \lambda_{t}$ and we compute the channel utilization $U=\operatorname{PDR} \times v_{0}$. We assume transmission power of $P=14 \mathrm{dBm}$. Figures 2 and 3 present the channel utilization based on $P D R_{I}, P D R_{D}$, and $P D R_{G}$ (the model by Georgiou and Raza [5]) as well as the corresponding value for unslotted ALOHA at SF12, for the distances $l_{0}=2500 \mathrm{~m}$ and $l_{0}=$ $7500 \mathrm{~m}$, respectively.

As for Aloha, we consider that a collision happens if another frame starts transmitting within $\tau_{j}$ before and after the start of a transmission. It is not the case in the model by Georgiou and Raza $[5,17]$. Their model rather corresponds to slotted Aloha or to continuous transmissions as in CDMA networks. Interestingly, probability $Q$ in this paper matches approximation $Q_{1}$ by Georgiou and Raza [5] with the precision of a few percent provided that we double the intensity of the interfering traffic in their expressions to account for the $2 \tau_{j}$ time during which at most one frame can start transmitting for no overlap. Consequently, in Figure 2 where $Q$ is the dominant cause of losses, the corrected $P D R_{G}$ curve closely matches our simulations and analytical expressions.

Moreover, in this short range case, the curves for $P D R_{I}$ and $P D R_{D}$ overlap, which is not the case for the longer range. We observe that for light offered traffic, all models roughly give the same results whereas differences become visible for higher offered traffic. Compared to all the models that take into account the capture effect, the unslotted ALOHA model [1] clearly stands out: physical capture increases the capacity of the network and channel utilization.

Simulations allow to analyze the effect of capture in case of multiple colliding frames: the crosses show the utilization when three overlapping frames are all dropped, whereas the rounds correspond to a more fine-grained analysis in which any frame can be received as long as it is received with a $\gamma$ margin over the sum of the power of the concurrent transmissions. For channel load below 1 Erlang, the assumption of a single interfering frame causes only a slight loss of precision.

\section{NUMERICAL RESULTS}

In this section, we present the results of the model and use it to optimize the SF boundaries. In this way, we obtain the number of nodes that a single gateway can serve, for various ranges and acceptable PDR. We compare the model with simulations that consider the effects of near-far co-SF and inter-SF interference. We highlight the necessity of power control in the SF7 zone and observe that inter-SF interference is moderate with our traffic model.
Table 3: SNR-based SF boundaries ( $\mathrm{km})$

\begin{tabular}{lrrrrrr} 
& $S F 7$ & SF8 & SF9 & SF10 & SF11 & SF12 \\
& $l_{5}$ & $l_{4}$ & $l_{3}$ & $l_{2}$ & $l_{1}$ & $l_{0}$ \\
\hline$H_{\text {target }}=99 \%$ & 1.18 & 1.43 & 1.72 & 2.07 & $\mathbf{2 . 4 1}$ & 2.82 \\
$H_{\text {target }}=90 \%$ & 2.23 & 2.68 & 3.23 & 3.89 & $\mathbf{4 . 5 4}$ & 5.23 \\
$H_{\text {target }}=70 \%$ & 3.09 & 3.72 & 4.48 & 5.40 & $\mathbf{6 . 3 0}$ & 7.36 \\
\hline \hline$\tau_{j}(\mathrm{~ms})$ & 102.7 & 184.8 & 328.7 & 616.5 & 1315 & 2466 \\
\hline
\end{tabular}

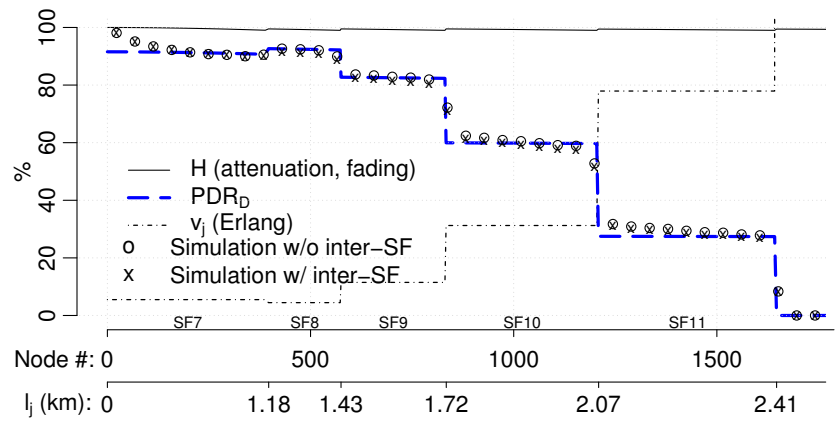

Figure 4: Small cell radius $\left(l_{1}=2.41 \mathrm{~km}\right)$, density of 90 nodes per $\mathrm{km}^{2}$, SNR-based SF boundaries. Attenuation (black curve) and collisions combine to give an estimated success probability (PDR). On the same scale, the dimensionless offered channel load $v_{j}$ is extremely small for the inner annuli and increases steeply for nodes located further away, where collisions are the dominant cause of packet loss. 1196 nodes within $2.07 \mathrm{~km}$ of the gateway experience a PDR above $60 \%$. Simulation corroborates the analysis, whether it takes into account inter-SF interference (crosses) or ignores it (circles).

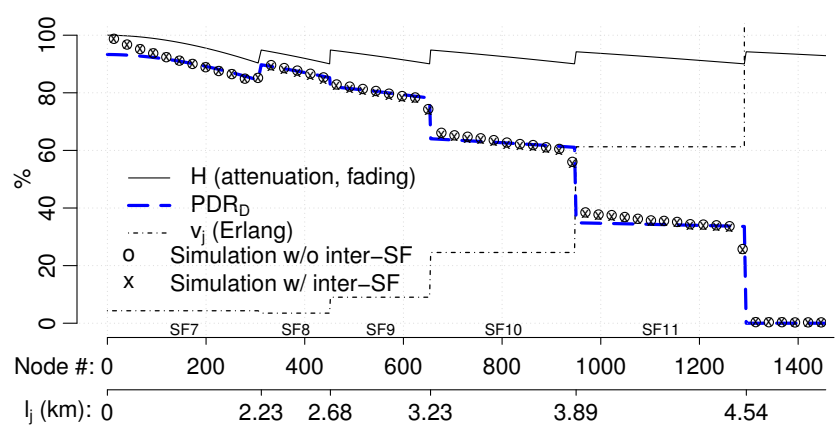

Figure 5: Medium cell radius $\left(l_{1}=4.54 \mathrm{~km}\right)$, density of 20 nodes per $\mathrm{km}^{2}$, SNR-based SF boundaries. Losses due to attenuation and fading start to weigh on PDR. 950 nodes within $3.9 \mathrm{~km}$ of the gateway experience PDR above $60 \%$

\subsection{SNR-based SF boundaries}

We start with the SF allocation and the annuli boundaries based on the measured SNR at the gateway, which corresponds to the value of probability $H$. The allocation reflects the operation of Adaptive Data Rate (ADR) in which the network server issues ADR commands to nodes indicating which SF to use. 


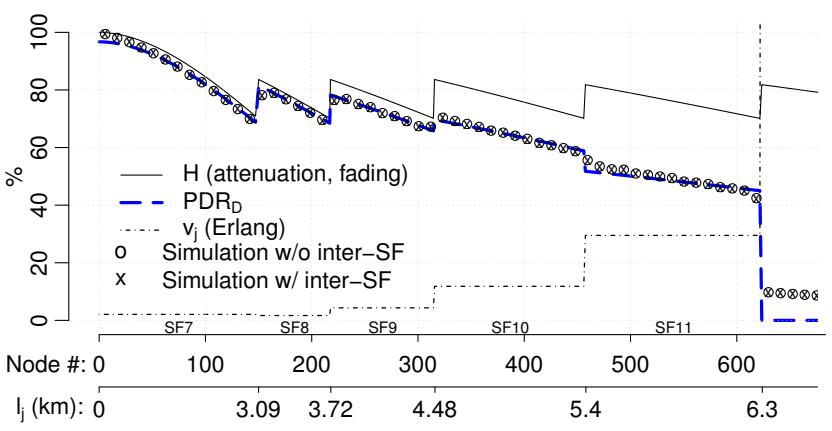

Figure 6: Large cell radius $\left(l_{1}=6.30 \mathrm{~km}\right)$, density of 5 nodes per $\mathbf{k m}^{2}$, SNR-based SF boundaries. For lower density, nodes using small SF face little contention, but collisions still impact PDR for nodes located further away. 443 nodes within $5.3 \mathrm{~km}$ of the gateway experience PDR above $60 \%$.

We consider three scenarios with three different radio ranges of $2.82,5.23$, and $7.36 \mathrm{~km}$ (small, medium, and large cell radius), respectively. For instance, in a medium range LoRaWAN cell, we would change SF each time the probability $H$ drops below $H_{\text {target }}=$ $90 \%$, which is the value of $H$ at a range of $5.23 \mathrm{~km}$. Solving Equation 4 for each $j$ gives the range for each data rate shown in Table 3 . For completeness, the table includes the $l_{0}$ distances at which $H$ drops below the target value at SF12, but the nodes beyond this radius cannot change to a lower SF and continue to use SF12. In the medium range case, the covered area is $86 \mathrm{~km}^{2}$, whereas the same gateway could cover an area of $170 \mathrm{~km}^{2}$ only at the cost of setting $H_{\text {target }}=70 \%$ (or a cell radius of $7.36 \mathrm{~km}$ ).

Figures 4, 5, and 6 present the values of $H, v_{j}$, and $P D R_{D}$ for the three cell sizes. For the small cell size, we consider the density of 90 nodes per $\mathrm{km}^{2}, 20$ nodes per $\mathrm{km}^{2}$ for the medium cell, and 5 per $\mathrm{km}^{2}$ for the large one. The $\mathrm{x}$-axis is the index of a node-we order the nodes by the distance to the gateway, e.g., the $1196^{\text {th }}$ node is at the distance of $2.07 \mathrm{~km}$ in the small cell.

The rounds and crosses represent the results obtained by simulation. The rounds give the average PDR for the transmissions emanating from the distance range on the $x$ axis under the assumption of perfect inter-SF orthogonality. The crosses correspond to simulation results that take into account inter-SF interference [15]. In the SF7 zone and especially for high node density, the simulation results diverge from the model because simulation takes into account the near-far problem: in case of collisions, nodes located close to the gateway always dominate the nodes further away.

All figures exhibit the same trend. Close to the gateway, for small SFs, there is little contention $\left(v_{j} \ll 1\right)$ and $P D R_{D}$ is satisfactory. We can also observe that the smaller the cell, the more nodes it can handle, because more nodes can use a lower SF and have a high $H$ probability. Shorter transmissions then reduce the channel load, which leaves room for incorporating many nodes before $P D R_{D}$ begins to drop due to collisions.

Conversely, in a larger cell, two adverse effects combine for the nodes located farther away. On the one hand, transmissions with higher SF take more airtime, resulting in more collisions. On the other hand, the annuli areas are larger going outward of the coverage disk, thus including more nodes at the same SF, which

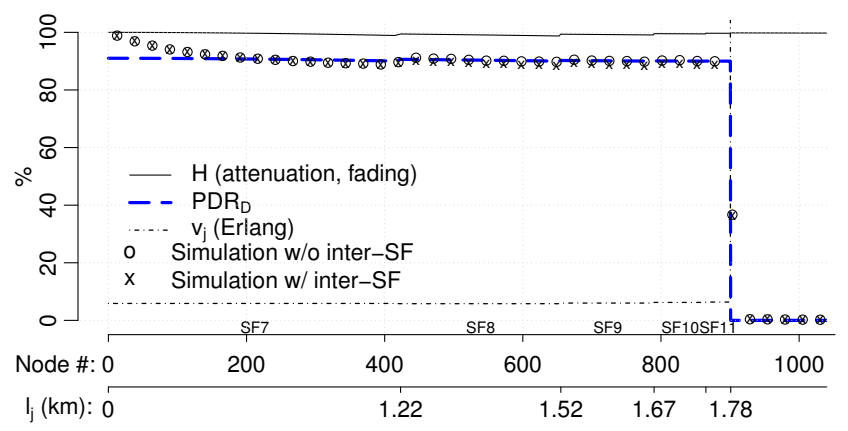

Figure 7: Small cell radius, density of 90 nodes per $\mathbf{k m}^{2}$, SF boundaries for target PDR of $\mathbf{9 0 \%}$.

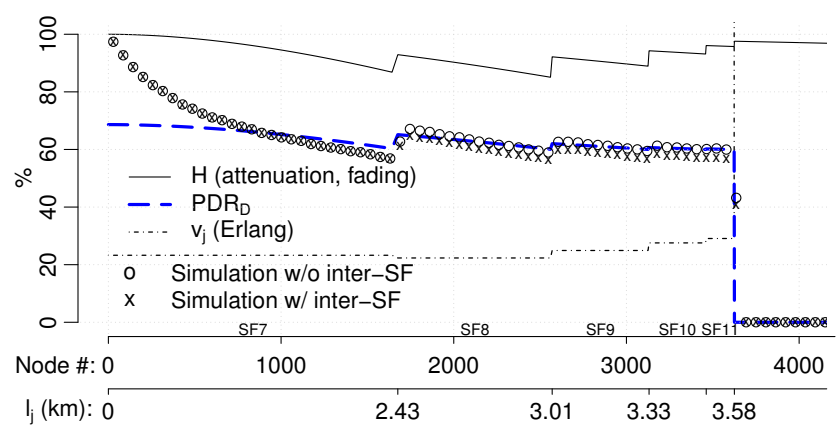

Figure 8: Small cell radius, density of 90 nodes per $\mathbf{k m}^{2}$, SF boundaries for target PDR of $60 \%$.

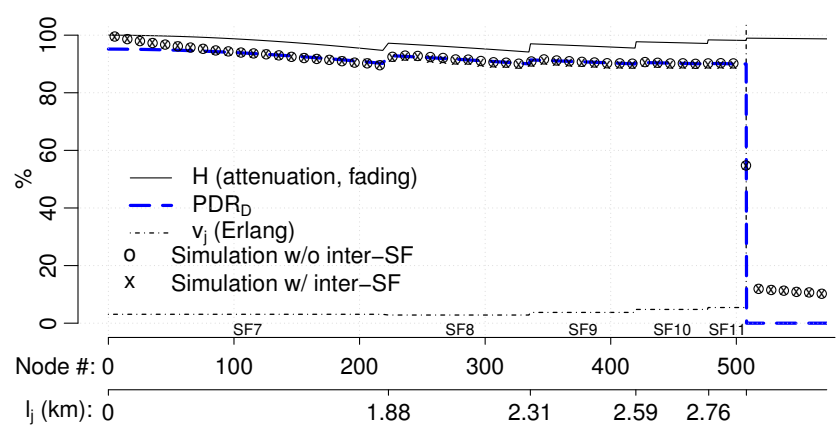

Figure 9: Medium cell radius, density of 20 nodes per $\mathbf{k m}^{2}$, SF boundaries for target PDR of $\mathbf{9 0 \%}$.

also leads to more collisions, and the overall node capacity drops to several hundreds of nodes when we want to get $60 \%$ PDR. The next section gives numerical examples of the number of nodes that a gateway can serve with optimized boundaries.

\subsection{PDR based boundaries and cell capacity}

We start by finding the SF boundaries to meet a given target $P D R_{D}$ : we use the bisection method to position the SF7 boundary at the distance where $P D R_{D}$ matches the target value. In this way, the annulus includes the maximal number of nodes that obtain at least target $P D R_{D}$. Then, we change to SF8 and increase its boundary to reach target $P D R_{D}$, and so on for other SF boundaries. 


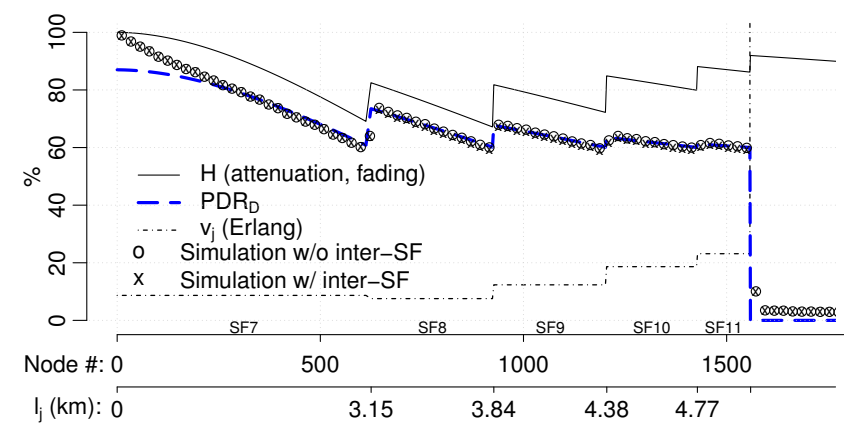

Figure 10: Medium cell radius, density of 20 nodes per $\mathbf{k m}^{2}$, SF boundaries for target PDR of $60 \%$.

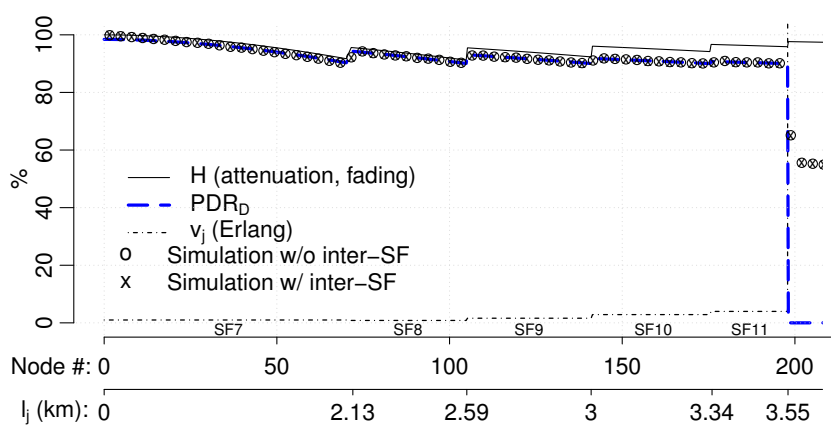

Figure 11: Large cell radius, density of 5 nodes per $\mathbf{k m}^{2}$, SF boundaries for target PDR of $\mathbf{9 0 \%}$.

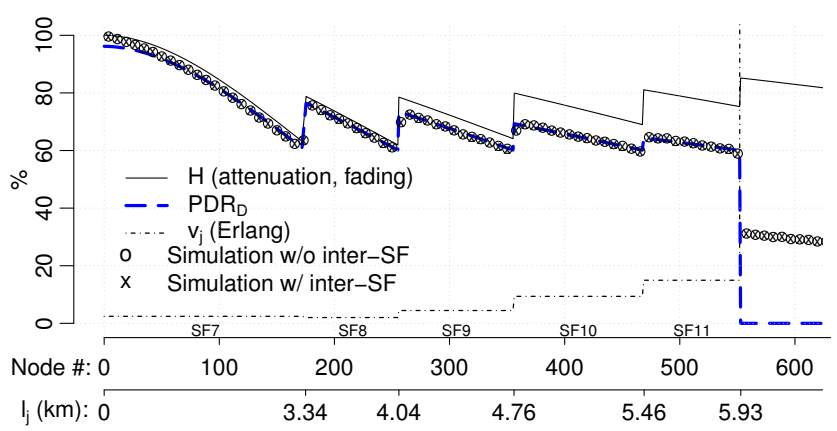

Figure 12: Large cell radius, density of 5 nodes per $\mathbf{k m}^{2}, \mathrm{SF}$ boundaries for target PDR of $60 \%$.

Figures 7, 8, 9, 10, 11, and 12 present $P D R_{D}$ for various densities of nodes and two target $P D R_{D}$ values of $60 \%$ and $90 \%$. In a small cell, probability $H$ is high, so nodes can easily attain $P D R_{D}$ of $90 \%$ (see Figure 7). Compared to Figure 4, we can observe that the frontiers for SF7 and SF8 are farther away by $50 \mathrm{~m}$ and $100 \mathrm{~m}$ respectively, but those for SF9, SF10, SF11 are closer to the gateway (by as much as $0.62 \mathrm{~km}$ for SF11), which results in a smaller cell with the total of 908 nodes with $P D R_{D}$ of $90 \%$. If we lower target $P D R_{D}$ to $60 \%$, the resulting cell is much larger with a significantly larger number of nodes benefiting from target $P D R_{D}: 3648$.

The model leaves aside inter-SF interference and near-far unfairness. In most cases, these effects have little impact, except for
Table 4: Maximal number of nodes with guaranteed PDR values of $60 \%$ and $90 \%$ for various node densities

\begin{tabular}{lccc} 
Nodes per $\mathrm{km}^{2}$ & Target PDR & $\begin{array}{c}\text { Number of } \\
\text { served nodes }\end{array}$ & $\begin{array}{l}\text { Coverage } \\
\text { radius } l_{1}(\mathrm{~km})\end{array}$ \\
\hline \multirow{2}{*}{90} & $90 \%$ & 908 & 1.79 \\
& $60 \%$ & 3648 & 3.59 \\
20 & $90 \%$ & 510 & 2.85 \\
& $60 \%$ & 1563 & 4.99 \\
5 & $90 \%$ & 198 & 3.56 \\
& $60 \%$ & 553 & 5.94 \\
\hline
\end{tabular}

heavily loaded small cells (see Figure 8 ) that we discuss in more detail in Sections 6.3 and 6.4.

Table 4 summarizes the capacity limits-the maximal number of nodes that benefit from a given level of target $P D R_{D}$. For small cells, optimizing the SF allocation has more effect on capacity, as the optimization process has more freedom to spread nodes between the SF annuli. In this case, there is more than a threefold difference between the maximal number of nodes that a single gateway can handle, with vs. without SF boundary optimization (e.g., 3648 vs. 1196 for $60 \%$ PDR).

When the cell range increases, optimization of SF allocation is more constrained and the maximal number of nodes is slightly less sensitive to the tuning of the SF boundaries. Moreover, the maximal number of nodes considerably increases when we lower target $P D R_{D}$ (e.g., to $60 \%$ ). This observation suggests that we can consider other ways of achieving high probability of successful packet delivery: nodes may repeat packets several times [18]. The resulting packet delivery probability is: $\operatorname{Pr}$ (packet delivery) $=1-$ $\left(1-P D R_{D}\right)^{n}$, where $n$ is the number of packet repetitions. For instance, for $P D R_{D}=60 \%$ and $n=3, \operatorname{Pr}$ (packet delivery) $=0.94$. Obviously, packet repetitions increase energy consumption; however, changing SF to larger values has a similar effect-going from $\mathrm{SF} j$ to $\mathrm{SF}\{j+1\}$ doubles the transmission duration. So, repeating 4 times a packet sent at SF7 has approximatively the same duration as a single transmission at SF9: the preamble overhead is the same because the preamble duration grows with SF and the only difference is that each symbol carries one more bit at each SF step. Using inter-frame error correction is even more effective, albeit more complex to implement [19].

In these calculations, we have considered an application that would saturate a band with a $1 \%$ duty cycle limit and wide enough to include 3 channels. In this case, an application generating one

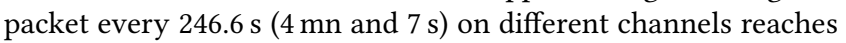
the duty cycle limit at SF12. Applications generating less traffic can either:

- send less packets, which saves energy for the individual nodes and reduces contention or, alternatively, increases the network capacity in terms of the number of nodes;

- repeat the data packets several times (packet repetition [18]) to improve the desired data delivery probability significantly.

\subsection{Power control in DR5 (SF7) zone}

In our model, all nodes in the same annulus experience a similar average channel gain. As the difference in the coding gain when changing from SF $j$ to $\mathrm{SF}\{j+1\}$ is approximately $2.5 \mathrm{~dB}$, the channel 


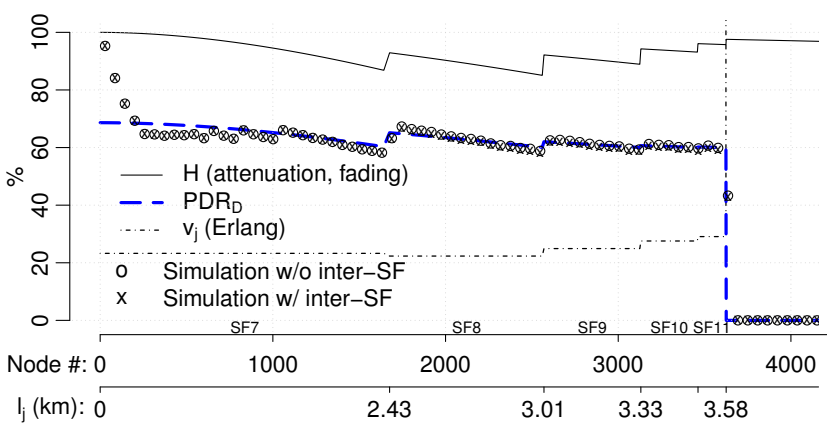

Figure 13: Small cell radius, density of 90 nodes per $\mathbf{k m}^{2}$, SF boundaries for target PDR of $60 \%$, power control in DR5 zone.

gains for a given SF are spread over this interval (i.e., $2.5 \mathrm{~dB}$ ), which is a quite reasonable assumption for all annuli except for the inner disk (DR5/SF7).

In this disk of radius $l_{5}$, channel gains are heterogeneous, so power control is necessary for fair access, as some nodes may have a markedly shorter distance to cross compared to others. Thus, a proper ADR scheme would make a node close to the edge of the SF7 zone use $P=14 \mathrm{dBm}$, as for higher SFs. It would then attribute the next lower transmission power to the nodes enjoying between $2 \mathrm{~dB}$ and $4 \mathrm{~dB}$ of average SNR advantage compared to the edge, and so on by steps of $2 \mathrm{~dB}$, until $P=2 \mathrm{dBm}$. This approach has two benefits, as illustrated in Figure 13 (to compare with Figure 8 presenting the results without power control): i) power-controlled nodes closer to the gateway are less harmful to farther nodes with respect to inter-SF interference and ii) energy consumption is marginally lower [20].

\subsection{Co-SF and inter-SF interference}

Experimental measurements by Mikhaylov et al. [21] showed that physical capture in presence of co-SF interference may happen well below $6 \mathrm{~dB}$ of the power margin. Compared to our model, this effect would tend to increase the actual packet delivery ratio. Some authors [16,21] also question the theoretical levels of inter-SF interference: in particular, low SFs may be jammed by other signals even when the power margin is of the order of $-6 \mathrm{~dB}$, whereas the expected values would be at least $10 \mathrm{~dB}$ lower [15]. This aspect is not overly critical: first, interference from higher SFs comes from nodes located farther away, so they face greater attenuation and thus, they are relatively harmless. Second, for the optimized SF boundaries, channel utilization is much reduced for higher SFs, so that nodes in the lower SF annuli face lower levels of accumulated interference from farther nodes compared to the SNR-based SF allocation for instance.

In fact, using the less favorable inter-SF rejection levels found by Croce et al. [16], inter-SF interference is non-negligible in a dense cell as we can see in Figure 14. However, since inter-SF interference mostly comes from transmissions of nodes located near the gateway, power control greatly helps (see Figure 15). In all cases, inter-SF interference is less of a problem with our homogeneous

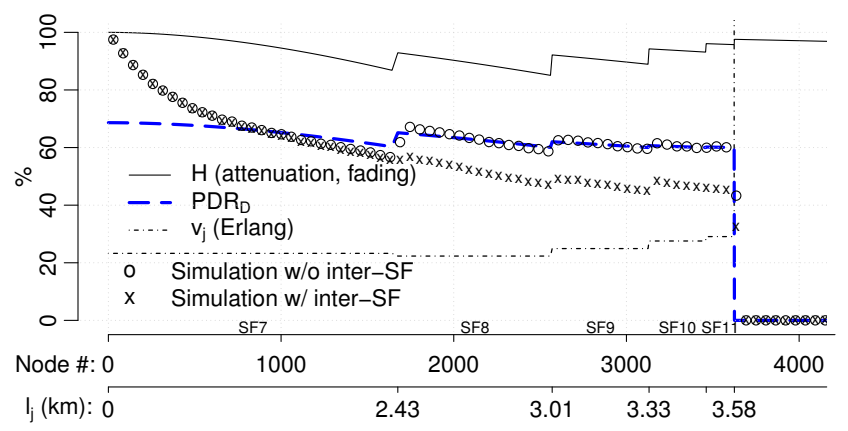

Figure 14: Small cell radius, density of 90 nodes per $\mathbf{k m}^{2}$, SF boundaries for target PDR of $60 \%$. Low inter-SF rejection [16].

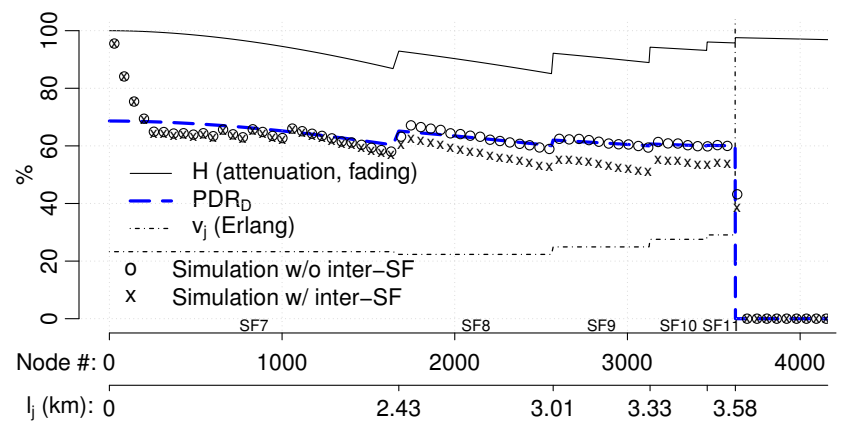

Figure 15: Small cell radius, density of 90 nodes per $\mathrm{km}^{2}$, SF boundaries for target PDR of $60 \%$. This is for the case of low inter-SF rejection and using power control in SF7 zone. Compared to Figure 14, PDR is more even among SF7 nodes and there is less (although still noticeable) inter-SF interference for SF8 and above.

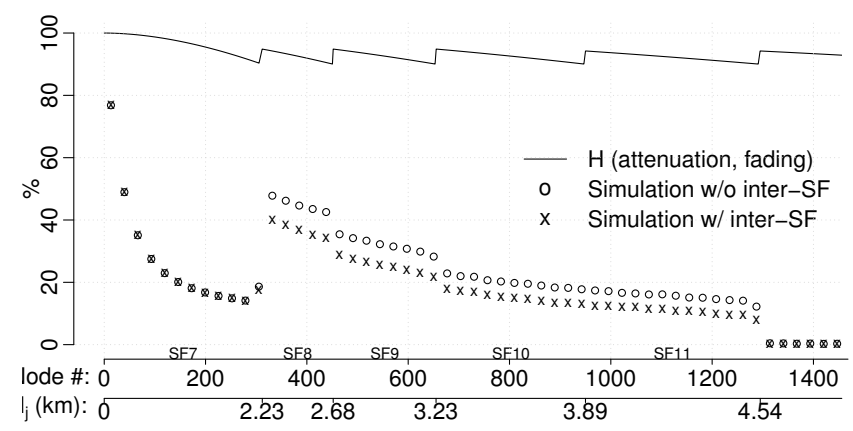

Figure 16: Medium cell radius, density of 20 nodes per $\mathbf{k m}^{2}$, SF boundaries for target SNR of $\mathbf{9 0 \%}$. In this simulation, all nodes use $1 \%$ duty cycle, which corresponds to the traffic model in most of the litterature $[5,6,10,17,22]$. Compared to Figure 5, a lot more packets are lost due to collisions and inter-SF interference still erase many transmissions.

traffic pattern, whereas it is exacerbated-as well as collisionswhen all nodes transmit using the same duty cycle as in the model by Georgiou and Raza presented in Figure 16. 


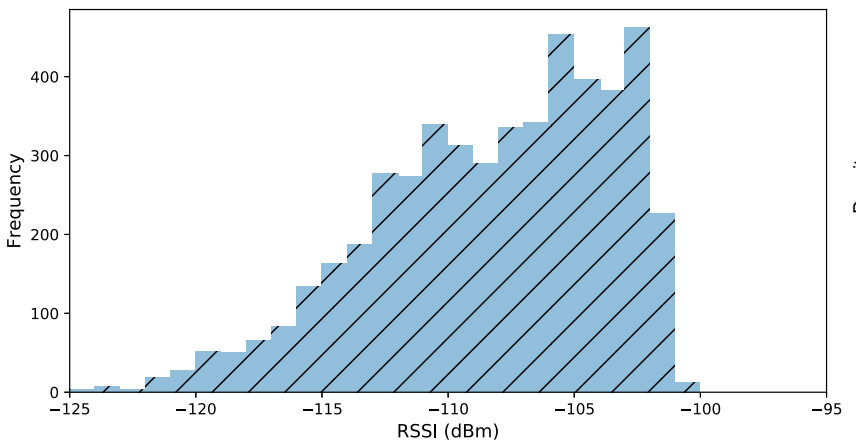

(a) $4.4 \mathrm{~km}$ link

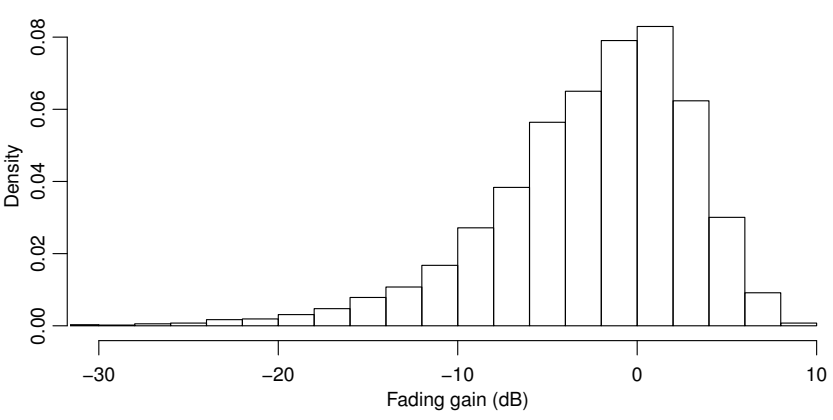

(b) Fading gain in Rayleigh channel

Figure 17: (a) Measured RSSI distribution for a $4.4 \mathrm{~km}$ LoRaWAN link from an outdoor sender, transmission power 14dBm, SF11. (b) Expected fading gain (in dB) distribution for a Rayleigh channel.

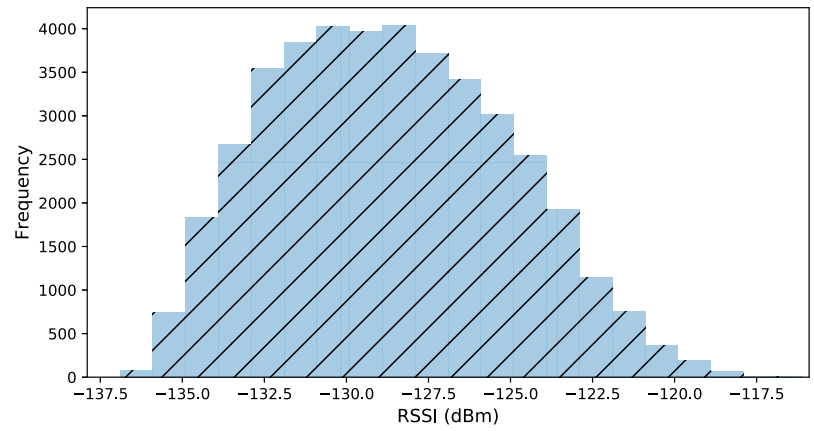

Figure 18: Measured RSSI distribution for a $2.4 \mathrm{~km} \mathrm{Lo-}$ RaWAN link, from an indoor sender, transmission power $14 \mathrm{dBm}, \mathrm{SF11}$. The distribution is truncated to the left because the reception power is then below the sensibility.

\section{EXPERIMENTAL ASSESSMENT OF THE CHANNEL MODEL}

The theoretical model presented in this paper as well as those in related work assume a Rayleigh fading channel. This assumption is quite usual, except in rare cases [23], but we draw attention to the fact that it was originally a model for mobile nodes. In the case of LPWAN networks, we consider mostly static nodes so that it would also be reasonable to assume less variability of the channel gain.

To get insight on how the channel gain behaves in the real-world, we have run long term experiments using an STMicroelectronics mote [24] and several gateways of The Things Network (TTN), a public LoRa network [25]. We have generated traffic and gathered statistics over 3 months to obtain the RSSI distributions of which two examples are shown in Figures 17 and 18. We send at SF11 with transmission power of $14 \mathrm{dBm}$. The transmissions alternate between 6 different frequency channels. Both histograms are for non-line of sight links in a suburban environment. The RSSI distribution of the long link (see Figure 17a) follows well the expected distribution of gains for a Rayleigh channel described in Section 2.1, which appears in Figure $17 \mathrm{~b}-$ deep fades of $-10 \mathrm{~dB}$ below the average gain $(0 \mathrm{~dB}$ in Figure 17b) are common.
For the longer link, we found that the use of several frequency channels explains the observed variability: the nodes are not mobile but frequency diversity has a similar effect, which is reminiscent of the reason why GSM networks use frequency hopping whereas GSM and LoRaWAN have similar transmission range, frequency channel separation, and carrier frequency.

The link in Figure 18 shows more attenuation because the sender is indoor, although the link in shorter. in this case, the variability would also randomize the outcome of the capture effect should a collision occur. Note that the sensitivity of approximately $-140 \mathrm{dBm}$ for SF11 truncates the distribution because the frames experiencing high fading are lost.

Finally, the measures confirm that the channel gains are always variable and that even for nodes with similar average channel gains, it would be very common to witness reception powers with a difference of $6 \mathrm{~dB}$. In this case, the capture effect allows the gateway to receive one of the colliding frames.

The Rayleigh channels assumption discussed in this section is important because a channel with less variability would dramatically change the results, with a longer range and also little inter-SF impact [23]. Experimentally, our finding is that the Rayleigh channel assumption is reasonable at least for links longer than a couple of $\mathrm{km}$.

\section{DISCUSSION AND RELATED WORK}

\subsection{Collision model}

Regarding collisions, we point out that solely meeting the capture condition (with probability $Q$ in Equation 7) is not sufficient to ensure successful reception, which is in contradiction with the model by Waret et al. [7]. We think that attenuation must always be taken into account, so PDR always integrates probability $H$ (Equation 4) in this paper.

Also, our model leads to a more conservative estimate of the probability of packet capture than those proposing to spread the energy of the overlapping interferer over the entire considered frame $[6,13,23]$.

We do not take into account the collision timing, although it has some effect [26] on the frame capture. We leave this refinement for future work. 


\subsection{Traffic models}

Many recent papers focusing on the LoRaWAN radio capacity assume that all nodes completely use their allowed duty cycle regardless of their SF $[5,6,10,17,22]$. This assumption means sending much more packets when a node is closer to the gateway, which is unrealistic: let us assume that an IoT application needs to send a packet every hour and the node is at the distance resulting in SF8. If the transmission conditions change and it can switch to SF7, it will not start sending packets every half an hour because of the possibility of doing so.

Under the assumption that all nodes use their allowed duty cycle like in Figure 16, if the metric for the capacity is the overall throughput, then the models give optimistic results (i.e., high throughput) because all nodes are able to fill up all available quasi-orthogonal sub-channels [6]. On the contrary, if the metric to express the capacity is the number of nodes $[5,10,17,22]$, the models result in dramatically small node capacity (e.g., 20 nodes for PDR of $80 \%$ ) because all nodes saturate their duty cycle, so there is little benefit in moving a node to a smaller SF, since it immediately transmits much more traffic and thus creates similar collision levels.

\section{ACKNOWLEDGEMENTS}

This work has been partially supported by the ANR projects: PERSYVAL-Lab under contract ANR-11-LABX-0025-01 and DiNS under contract ANR-19-CE25-0009-01.

\section{CONCLUSION}

In this paper, we propose a model for the capacity of a LoRaWAN cell that takes into account collisions and the capture effect. It allows to assess the number of nodes that can share a single LoRaWAN cell for a given node density, cell range, traffic generation rate, and target PDR. We have validated the model with discrete event simulations.

Our results show that a LoRaWAN cell can handle thousands of devices. We also consider the effect of inter-SF interference, which turns out to be relatively small: interference from higher SFs comes from nodes located farther away, so they face greater attenuation and thus, they do not interfere with lower SF nodes. Moreover, power control can greatly help to avoid the jamming of distant nodes by the near ones.

Our hypothesis of similar traffic intensity in the cell puts under the spotlight the problem of suitable SF allocation. In particular, for devices close to the gateway, it is critical to distribute as much traffic as possible on lower SFs. For farther devices, there is a balance to find: they need to use larger SFs to reduce the impact of fading and at the same time, use the smallest possible SFs to avoid collisions.

Finally, a salient question left for future work is how to practically allocate SFs: gateways have limited downlink transmission opportunities [27], so that a practical ADR control method would have to take this limitation into account. Besides, a real network also has to consider the presence of multiple gateways and hence, macro-diversity reception, a central feature of LoRaWAN, which we plan to analyze in the future.

\section{REFERENCES}

[1] L. Kleinrock and F. Tobagi. Packet Switching in Radio Channels: Part I - Carrier Sense Multiple-Access Modes and Their Throughput-Delay Characteristics. IEEE
Transactions on Communications, 23(12), 1975

[2] A. Kochut, A. Vasan, A. Udaya Shankar, and A. K. Agrawala. Sniffing Out the Correct Physical Layer Capture Model in 802.11b. In IEEE ICNP, Berlin, Germany, 2004.

[3] J. Haxhibeqiri, F. Van Den Abeele, I. Moerman, and J. Hoebeke. LoRa Scalability: A Simulation Model Based on Interference Measurements. Sensors, 17(6):1193, 2017.

[4] R. Fernandes, R. Oliveira, M. Luís, and S. Sargento. On the real capacity of LoRa networks: The impact of non-destructive communications. IEEE Communications Letters, 23(12):2437-2441, 2019.

[5] O. Georgiou and U. Raza. Low Power Wide Area Network Analysis: Can LoRa Scale? IEEE Wireless Communications Letters, 6(2), April 2017.

[6] Z. Li, S. Zozor, J.-M. Brossier, N. Varsier, and Q. Lampin. 2D Time-Frequency Interference Modelling Using Stochastic Geometry for Performance Evaluation in Low-Power Wide-Area Networks. In IEEE International Conference on Communications (ICC), May 2017.

[7] A. Waret, M. Kaneko, A. Guitton, and N. El Rachkidy. LoRa Throughput Analysis with Imperfect Spreading Factor Orthogonality. IEEE Wireless Communications Letters, 8(2):408-411, 2019.

[8] J. Petäjäjärvi, K. Mikhaylov, M. Pettissalo, J. Janhunen, and J. Iinatti. Performance of a Low-Power Wide-Area Network Based on LoRa Technology: Doppler Robustness, Scalability, and Coverage. International fournal of Distributed Sensor Networks, 13(3), 2017

[9] K. Mikhaylov, J. Petäjäjärvi, and T. Hänninen. Analysis of Capacity and Scalability of the LoRa Low Power Wide Area Network Technology. In 22th European Wireless Conference, May 2016.

[10] A. Mahmood, E. Sisinni, L. Guntupalli, R. Rondon, S. A. Hassan, and M. Gidlund. Scalability Analysis of a LoRa Network under Imperfect Orthogonality. IEEE Transactions on Industrial Informatics, 15(3):1425-1436, 2019.

[11] Semtech. SX1272/73 - $860 \mathrm{MHz}$ to $1020 \mathrm{MHz}$ Low Power Long Range Transceiver, 2017. URL https://www.semtech.com/uploads/documents/sx1272.pdf.

[12] D. Bankov, E. Khorov, and A. Lyakhov. Mathematical Model of LoRaWAN Channel Access with Capture Effect. In 28th IEEE Annual International Symposium on Personal, Indoor, and Mobile Radio Communications, PIMRC, Oct 2017.

[13] D. Magrin, M. Centenaro, and L. Vangelista. Performance Evaluation of LoRa Networks in a Smart City Scenario. In IEEE International Conference on Communications (ICC), May 2017.

[14] S. Demetri, M. Zúñiga, G. P. Picco, F. Kuipers, L. Bruzzone, and T. Telkamp. Automated estimation of link quality for LoRa: A remote sensing approach. In 18th International Conference on Information Processing in Sensor Networks, (ACM IPSN), pages 145-156, 2019.

[15] C. Goursaud and J.-M. Gorce. Dedicated Networks for IoT: PHY/MAC State of the Art and Challenges. EAI Endorsed Transactions on Internet of Things, 1(1), 10 2015.

[16] D. Croce, M. Gucciardo, S. Mangione, G. Santaromita, and I. Tinnirello. Impact of LoRa Imperfect Orthogonality: Analysis of Link-Level Performance. IEEE Communications Letters, 22(4):796-799, 2018.

[17] O. Georgiou, C. Psomas, and I. Krikidis. Coverage scalability analysis of multi-cell LoRa networks. In IEEE International Conference on Communications (ICC), June 2020.

[18] Y. Birk and Y. Keren. Judicious Use of Redundant Transmissions in Multichannel ALOHA Networks with Deadlines. IEEE Journal on Selected Areas in Communications, 17(2):257-269, 1999

[19] U. Coutaud, M. Heusse, and B. Tourancheau. Fragmentation and forward error correction for LoRaWAN small MTU networks. In MaDeLoRa workshop, Embedded Wireless Systems and Networks (EWSN). ACM, 2020.

[20] M. Nunez Ochoa, A. Guizar, M. Maman, and A. Duda. Evaluating LoRa Energy Efficiency for Adaptive Networks: From Star to Mesh Topologies. In 13th IEEE International Conference on Wireless and Mobile Computing, Networking and Communications (WiMob'17), October 2017

[21] K. Mikhaylov, J. Petäjäjärvi, and J. Janhunen. On LoRaWAN Scalability: Empirical Evaluation of Susceptibility to Inter-Network Interference. In 2017 European Conference on Networks and Communications (EuCNC), June 2017.

[22] A. Hoeller, R. D. Souza, O. L. Alcaraz López, H. Alves, M. de Noronha Neto, and G. Brante. Analysis and Performance Optimization of LoRa Networks With Time and Antenna Diversity. IEEE Access, 6, 2018.

[23] J. Markkula, K. Mikhaylov, and J. Haapola. Simulating LoRaWAN: On Importance of Inter Spreading Factor Interference and Collision Effect. In IEEE International Conference on Communications (ICC), 2019.

[24] STMicroelectronics. B-L072Z-LRWAN1. www.st.com, 2018.

[25] W. Giezeman and J. Stokking. The Things Network. URL https://www. thethingsnetwork.org.

[26] A. Rahmadhani and F. Kuipers. When LoRaWAN Frames Collide. In 12th International Workshop on Wireless Network Testbeds, Experimental Evaluation \& Characterization, WiNTECH '18. ACM, 2018.

[27] V. Di Vincenzo, M. Heusse, and B. Tourancheau. Improving Downlink Scalability in LoRaWAN. In IEEE International Conference on Communications (ICC), Shanghai, China, May 2019. 